\title{
COMENTARIO A "¿QUÉ SON LAS LEYES \\ DE LA NATURALEZA?" \\ DE VAN FRAASSEN
}

\author{
León OLIVÉ \\ InstrTuto de InVestigaciones Filosóficas \\ Universidad Nacional autónoma de MExico
}

En este comentario me concentraré en la cuestión que es obviamente la más controvertible y central del trabajo de Bas van Fraassen: la pretensión de que no hay leyes de la naturaleza, o por lo menos de que lo mejor que podemos hacer es suponer que no las hay.

Debe aclararse desde el principio que, ciertamente, si uno quiere utilizar el argumento de la ciencia para enfatizar la importancia de la discusión de la noción de ley de la naturaleza, se debería tratar de probar la existencia de las leyes por medio de argumentos independientes con respecto al de la ciencia. Pero aquí sugeriré que una teoría de las leyes y una tcoría de la ciencia están más entretejidas, y dependen entre sí, más de lo que van Fraassen parece aceptar. Sostendré que la creencia en las leyes de la naturaleza es necesaria para una teoría que haga inteligible a la ciencia como un proceso racional, y que en efecto este requisito es lo suficientemente fuerte para llevarnos a la creencia básica en las leyes de la naturaleza. Pero debo conceder que la concepción de lo que son las leyes de la naturaleza debe ser previa lógicamente al análisis de la ciencia propiamente dicho.

Deseo aclarar que por mi parte no comparto la creencia de que la ciencia es primordialmente un proceso de descubrimiento de leyes. Esto no significa negar que, comoquiera que se tomen las leyes y sus nociones correspondientes, éstas juegan un papel fundamental en las teorias científicas. Pero uno debe tener en mente la distinción entre las teorias cientificas y una noción más amplia de ciencia, como el mismo van Fraas. sen lo reconoce, la cual debe incluir la noción de las actividades científicas con ciertos propósitos. Sostendré que la noción de ley natural se requiere para hacernos inteligibles esas actividades y esos propósitos.

Es verdad que no todo lo que cuenta como una explicación científica recurre a la noción de ley. Esto debe concederse. Ahora bien, lo que sostengo es que la ciencia no es primordialmente una empresa en busca 
de regularidades ni en busca de explicaciones de ellas, pero que sin embargo es muy importante para la ciencia recurrir a esas regularidades para hacer predicciones y para conseguir una intervención efectiva en el mundo. En esos casos, ciertamente existe una creencia en la persistencia en el futuro del mismo patrón de eventos. El problema es si existen fundamentos para creer que las regularidades tienen $q u e$ exhibir el mismo patrón. Los que creen en las leyes responden que estas regularidades tienen que, si son instancias de una ley. Van Fraassen desea convencernos de que no se pierde nada importante al creer que las regularidades pueden muy bien existir sin que haya ninguna razón para ello. Ciertamente, debemos creer que hay regularidades que no tienen ninguna razón de ser, es decir, que no hay leyes que nos permitan explicarlas. Y no puede verse como irracional el tener la expectativa de que una regularidad que hasta ahora ha subsistido, y que puede ser explotada por los seres humanos, persistirá en el futuro. Así, van Fraassen tiene razón al decir que no es un escéptico acerca del futuro, y que tampoco es irracional. En efecto, es tan racional como cualquier otra persona en cualquier sociedad que se las arregla para sobrevivir durante un periodo. De hecho, creo que van Fraassen está hablando acerca de lo que yo llamaría una tecnología precientífica y de las creencias y el conocimiento que tal tecnología involucra. Esto es, la distinción entre ciencia y tecnología, si es que para van Fraassen hay alguna, se vuelve sólo una diferencia en el grado de sofisticación de las teorías. ¿Pero por qué deberíamos tener teorías más sofisticadas? De acuerdo con van Fraassen, la ciencia, o la tecnologia, que en realidad viene a ser lo mismo, deberían preocuparse únicamente por la identificación de regularidades, y por nuestra parte simplemente deberíamos tener esperanza en que ocurrirá lo mejor, es decir, que persistirán.

Por mi parte, quiero insistir en que la suposición de que si hay leyes de la naturaleza explica por qué la tecnología es exitosa, aun cuando los modelos tecnológicos propiamente dichos pueden no contener leyes científicas. Esto es decir que el supuesto que no se hace explícito, y que en muchos casos no puede ser accesible para los usuarios de la tecnologia, es que hay efectivamente algo en la naturaleza responsable de la ocurrencia de los eventos, y que la explicación de las regularidades debe referirse a ese agente responsable.

Así, debe concederse que no es irracional tener la expectativa de que las regularidades persistirán; sin embargo, existe una gran diferencia entre terier esa expectativa sin fundamento alguno (aunque nos resistamos a llamar irracional a esa expectativa en virtud de que tenemos otras razones de orden pragmático) y tener una expectativa fundamentada racionalmente. 
En suma, sostengo que al asumir que hay leyes de la naturaleza ganamos en el poder explicativo de nuestra teoria de la ciencia. También ganamos en la posibilidad de comprender que no sólo por razones pragmáticas es racional hacer inferencias, y también podemos dotar de mayor sentido a la idea de la inferencia a la mejor explicación. Más aún, ganamos un criterio para comparar explicaciones; con esto podemos entender mejor la noción de inferencia a la mejor explicación.

Me parece que todo esto es el resultado del argumento de Armstrong en este respecto. Una pequeña digresión es adecuada en este momento, pues la razón que van Fraassen aduce para rechazar el argumento de Armstrong es que en vez de llegar a la conclusión de que hay leyes de la naturaleza, parte de la premisa de que tales leyes existen. Concedido. Pero creo que la estrategia argumentativa de Armstrong es precisamente la de partir de esa premisa y obtener la serie de consecuencias favorables a las que me referí arriba, mientras que van Fraassen parece simplemente querer rechazar ese supuesto. En este momento me parece apropiado señalar que no encuentro en el trabajo de van Fraassen una refutación convincente del argumento de Armstrong. Lo que van Fraassen dice en la página 255 no es suficientemente fuerte. De acuerdo con su ejemplo $H$ puede ser la única explicación que hasta ahora ha ofrecido la ciencia para explicar la evidencia $E$. Debe reconocerse que es posible que tal explicación sea falsa (tanto como que es posible que sea verdadera). Pero van Fraaseñ jüega aquí con una ambigüedad del término "mejor". En el ejemplo de van Fraassen $H$ es la mejor explicación disponible porque por hipótesis no hay otra explicación a la mano, mientras que el argumento de Armstrong es que la mejor explicación es aquella que recurre a leyes. Aquí sólo deseo señalar que la propuesta de Armstrong no ha sido derrotada.

Lo que he sugerido hasta aquí es que el supuesto de que hay leyes de la naturaleza nos permite tener una teoría racional de la ciencia y de la tecnología, así como una manera racional de entender la inferencia a la mejor explicación y la inducción.

Pasemos ahora al punto crucial del trabajo de van Fraassen: la pretensión de que es imposible dotar de algún sentido a la siguiente "parte inalienable del concepto de ley" (para usar sus propias palabras; p. 257), a saber, que "es posible explicar los fenómenos diciendo que son como son porque deben ser de esa manera, y que deben ser de esa manera en virtud de una ley de la naturaleza" (p. 257).

Es efectivamente imposible dotar de algún sentido a esta proposición mientras se mantenga que la realidad está constituida únicamente por sucesiones de eventos y mientras mantengamos a las regularidades como punto de partida para la construcción de nuestra noción de ley de la 
naturaleza. Lo anterior conduce a análisis lógicos y lingüísticos en donde las leyes se suponen descritas adecuadamente por medio de enunciados de la forma "todos los que sean $F$ deben ser $G$ ", o peor aún, "debe ser el caso que todos los que sean $F$ sean $G$ ".

Para ver más claramente lo que quiero decir comparemos los siguientes puntos de vista:

F) No tengo razón alguna para esperar que la regularidad subsistirá en el futuro, porque no hay razones en la naturaleza para que persista; sin embargo, es racional que yo espere que subsistirá. $\mathrm{Y}$ aquí sólo es posible recurrir a razones pragmáticas para fundamentar lo que es racional.

Ahora consideremos la siguiente alternativa:

R) La razón que tengo para esperar que la regularidad subsistirá es que hay mecanismos perdurables en la naturaleza tales que, cuando se activan en circunstancias apropiadas, producen, es decir, son responsables de la ocurrencia de la regularidad de los eventos que observamos.

Este es, por supuesto, un compromiso metafísico muy fuerte que van Fraassen rechazaría. Lo que me importa destacar es que tendríamos que examinar los dos puntos de vista rivales para darnos cuenta de qué se logra con cada uno de ellos.

El punto de vista alternativo al de van Fraassen debe ir acompañado de una concepción de la ciencia que no considere que ésta busca primordialmente regularidades, ni siquiera leyes, sino cosas. Se debe considerar que la ciencia se interesa por descubrir la manera en que las cosas están estructuradas y la forma en que tienden a actuar. En el proceso se descubren leyes de la naturaleza, es decir, la manera en que las cosas tienden a actuar, esto es, cuál es la clase de fenómenos y de regularidades que las cosas producen a través de sus actividades eficientes. De esta manera es posible explicar las regularidades e incluso en ocasiones manipularlas.

¿Qué podemos decir ahora de "la parte inalienable del concepto de ley"? En efecto, se pueden explicar los fenómenos diciendo que son como son porque deben ser de esa manera, y deben ser de esa manera en virtud de una ley de la naturaleza. Pero ahora esto quiere decir que hay un objeto en la naturaleza, un mecanismo - pensémoslo como un sistema-, los estados sucesivos del cual son los eventos que pueden por ejemplo dar lugar a las regularidades que observamos normalmente. Lo que dice una ley es que se producirá una regularidad si hay un mecanismo de esa clase, el cual no se ve impedido para actuar de la manera en que tiende a hacerlo, por algunos otros factores, y que la manera en que tiende a actuar se debe a su estructura. De lo anterior se sigue que las leyes de la naturaleza en general no quedarán correctamente descritas 
por enunciados de la forma "todos los que son $F$ son $G$ ", o "debe ser el caso que todos los que son $F$ son $G$ ", sino que se requieren enunciados singulares que describan universales reales.

Me parece que de esta manera podemos dotar de sentido a aquello que van Fraassen alega que no puede tenerlo ("la parte inalienable del concepto de ley"), pero también podemos descartar entonces la objeción que van Fraassen presenta contra Armstrong (p. 242). En efecto, van Fraassen muestra de una manera muy ingeniosa que de acuerdo con la propuesta de Armstrong " $F$ necesita de $G$ " puede ser verdadera, mientras que algún particular $F$ puede no ser un $G$, esto es, la ley no implica la regularidad correspondiente. Pero para el punto de vista que aquí he sugerido, ésta no es realmente una objeción, pues esta perspectiva no queda comprometida con la implicación en cuestión, y, más aún, puede explicar por qué algunas veces alguna ley puede estar actuando sin que de hecho ocurra la regularidad correspondiente.

Es contingente que el mundo sea como es. Así, no creo que tenga sentido sostener el punto de vista de que es necesario que tal y tal sea el caso, si no se añade alguna otra clarificación. Pero tiene sentido mantener el punto de vista de que dado que el mundo es como es, necesariamente se producirán ciertas regularidades si están presentes los mecanismos apropiados y éstos pueden actuar sin que haya poderes que los contrarresten. Ahora bien, debe considerarse que la pregunta de por qué es el mundo como es y no de manera diferente, pertenece a una mala metafísica. Así que podemos detenernos en este momento: cuando tenemos una idea racional de las regularidades y de nuestras creencias en ellas y, más aún, podemos explicar por qué la ciencia y la tecnología son exitosas en gran medida y por qué nosotros somos racionales al tener esas creencias.

En conclusión, el supuesto de que no hay leyes de la naturaleza parece ser bueno, en principio, si se acepta una ontología que incluya únicamente regularidades y sucesiones de eventos en el mundo real. No debe sorprender que bajo este supuesto la noción de ley de la naturaleza se vuelva ininteligible, y así parece un movimiento natural y comprensible el descartar esta noción y sostener en cambio la idea de que las leyes cientificas son únicamente instrumentos de utilidad lingüistica o teórica. El precio que hay que pagar por esta posición no es sólo la ininteligibilidad de la noción de ley de la naturaleza, sino también lo siguiente:

a) Una pobre idea de ciencia, pues debe considerarse que ésta busca primordialmente un registro de regularidades.

b) Sólo puede haber razones pragmáticas para sostener que la inferencia inductiva es, después de todo, racional. Pero debe concederse que no hay razones para esperar que las regularidades subsistirán. 
c) No puede haber una distinción significativa entre ciencia y tecnología. Más aún, es un milagro que la ciencia se haya desarrollado a partir de la pura tecnología, y es un milagro que ambas sean exitosas. Sin embargo, todavia podría sostenerse que somos racionales al creer en milagros.

d) Se excluye de la explicación de la ciencia y de cómo ésta es posible, la idea que tengamos de cómo es el mundo.

e) Es difícil dotar de sentido a la actividad de la construcción de modelos tal como proceden normalmente los científicos.

Por otro lado, si adoptamos la creencia que puede justificarse racionalmente de que hay leyes de la naturaleza, encontramos las siguientes ventajas:

a) Puede verse que la ciencia es una empresa racional, que no busca primordialmente leyes, pero que sin embargo constantemente descubre algunas. Esto explica por qué es exitosa al predecir y por qué tiene bases racionales para sus pretensiones de producción y de éxito.

b) Es posible concebir cuáles son las condiciones de posibilidad de la ciencia y de la tecnología sobre la base de que de hecho éstas existen, y cabe preguntarse sobre la manera en que el mundo debe ser para que la ciencia y la tecnología sean posibles.

c) Es posible distinguir a la ciencia de la tecnología y, más aún, la primera puede explicar a la segunda.

d) La noción de inferencia a la mejor explicación puede ser claramente concebida y puede mostrarse que está racionalmente fundamentada.

e) Puede mostrarse que las inferencias inductivas son racionales sobre la base de razones epistémicas, y no sólo por razones pragmáticas.

f) La actividad de construcción de modelos se vuelve racional y bien fundada.

Para concluir, es preciso apuntar que la gran diferencia entre las posiciones que aquí se han comentado comienza con la riqueza de la ontología que cada una está dispuesta a aceptar. ¿Pero por qué deberíamos sentir una inclinación por la más simple? Van Fraasen todavía tiene que ofrecernos razones convincentes para que asumamos este valor. 